\section{POSSIBILIDADES DE ATUAÇÃO PROFISSIONAL DO PSICÓLOGO NO ÂMBITO DA ATENÇÃO BÁSICA EM SAÚDE}

\author{
Possibilities of professional performance of the psychologist in \\ the Primary Health Care context
}

\author{
Posibilidades de actuación profesional del psicólogo en el \\ ámbito de la atención básica de salud
}

\section{RESUMO}

Objetivo: O presente trabalho objetivou identificar, nas evidências científicas, as atividades que caracterizam a atuação do psicólogo no contexto da Atenção Básica de Saúde. Métodos: Trata-se de uma revisão sistemática, realizada entre os meses de agosto a dezembro de 2015, de acordo com as diretrizes do protocolo PRISMA. Foram utilizados os descritores "Psicologia", "Atuação" e "Atenção Básica" na Biblioteca Virtual de Saúde. Adotou-se como estratégia a busca direta, utilizando as palavras-chave "Psicologia na Atenção Básica" nas bases de dados SciELO, LILACS e Index Psi Periódicos Técnico-Científicos. Os critérios de elegibilidade foram: a) veículo de publicação: periódicos indexados; b) período de publicação: 2010-2014; c) idiomas: português, inglês ou espanhol; d) modalidade da produção: estudos empíricos, relatos de experiências, estudos de casos; e) qualidade metodológica: pesquisas que definissem precisamente quais eram seus participantes, instrumentos, procedimentos e tipo de estudo. A busca pelos artigos ocorreu a partir de uma análise minuciosa dos resumos das pesquisas encontradas, consistindo numa procura de estudos relacionados à atuação do psicólogo na Atenção Básica e que se enquadrassem nos critérios de elegibilidade previamente determinados. Resultados: A princípio, encontrou-se um total de 107 artigos, contudo, após a leitura dos resumos, chegou-se ao total de 11, os quais foram lidos completamente. Os dados indicaram o aparecimento de duas formas mais amplas de atuação do psicólogo na Atenção Básica: atuação na Estratégia de Saúde da Família e como profissional do Núcleo de Apoio à Saúde da Família. Conclusão: Apesar dos inúmeros desafios cotidianos, é perceptível a concretização de práticas que levem em consideração tanto aspectos referentes à subjetividade quanto sócio-histórico-econômico-político-culturais.

Descritores: Psicologia; Atuação; Psicologia; Atenção Primária à Saúde.

\section{ABSTRACT}

Objective: This study aimed at identifying, within the scientific evidences, the activities that characterize the psychologist's performance in the Primary Health Care context. Methods: This is a systematic review, carried out between August and December 2015, in accordance with PRISMA protocol guidelines. "Psychology", "Performance" and "Primary Health Care" were used as descriptors in the Virtual Health Library. Direct search using the keywords "Psychology in Primary Health Care" was the strategy adopted in the databases SciELO, LILACS and Index Psi Technical-Scientific Journals. The eligibility criteria were: a) publishing vehicles: indexed journals; b) publishing period: 2010-2014; c) language: Portuguese, English or Spanish; d) study type: empirical studies, experience reports, case studies; e) methodological quality: researches that precisely define their participants, instruments, procedures and study type. The search for articles was conducted following a thorough analysis of the abstracts of the retrieved researches, consisting in a search for studies related to the psychologist's performance in the Primary Health Care context and that met the previously established eligibility criteria. Results: A total of 107 articles was initially found but, after reading the abstracts, the number reached a total of 11, whose full texts
Artigo de Revisão
1) Universidade Federal do Piauí - UFPI Teresina (PI) - Brasil

2) Instituto Federal do Maranhão (IFMA) São Luis (MA) - Brasil
Recebido em: 24/12/2015

Revisado em: 11/03/2016

Aceito em: 30/03/2016 
were read. The data indicated the emergence of two broader forms of psychologist's performance within the Primary Health Care: performance in the Family Health Strategy and as a professional of the Family Health Support Center. Conclusion: Despite the several daily challenges, it is noticeable the implementation of practices that take into account the aspects related to the subjectivity, as well as the social, historical, economic, political and cultural aspects.

Descriptors: Psychology; Acting out; Primary Health Care.

\section{RESUMEN}

Objetivo: El objetivo del presente trabajo fue identificar en las evidencias científicas las actividades que caracterizan la actuación del psicólogo en el contexto de la Atención Básica de Salud. Métodos: Se trata de una revisión sistemática realizada entre los meses de agosto y diciembre de 2015 según las directrices del protocolo PRISMA. Se utilizó los descriptores "Psicología", "Actuación" y "Atención Básica" en la Biblioteca Virtual de Salud. Se adoptó la búsqueda directa como estrategia utilizando las palabras-clave "Psicología de la Atención Básica" en las bases de datos SciELO, LILACS y el Index Psi Periódicos TécnicoCientíficos. Los criterios de elegibilidad fueron: a) el modo de publicación: periódicos indexados; b) el periodo de publicación: 2010-2014; c) los idiomas: portugués, inglés o español; d) la modalidad de la producción: estudios empíricos, relatos de experiencias, estudios de casos; e) la calidad metodológica: investigaciones que definieron sus participantes, instrumentos, procedimientos y el tipo del estudio con precisión. La búsqueda de los artículos se dio a partir de un análisis minucioso de los resúmenes de las investigaciones encontradas a través de una búsqueda de estudios relacionados a la actuación del psicólogo de la Atención Básica y que se encuadraran con los criterios de elegibilidad determinados previamente. Resultados: En principio se encontró un total de 107 artículos, sin embargo, después de la lectura de los resúmenes fueron elegidos 11 artículos de los cuales se realizó la lectura completa. Los datos indicaron dos formas amplias de actuación del psicólogo de la Atención Básica: la actuación en la Estrategia de Salud de la Familia y como profesional del Núcleo de Apoyo a la Salud de la Familia. Conclusión: Aunque haya inúmeros desafios del cotidiano es perceptible la concretización de prácticas que consideran los aspectos de la subjetividad y los socio-histórico-económicopolítico-culturales.

Descriptores: Psicología; Actuación (Psicología); Atención Primaria de Salud.

\section{INTRODUÇÃO}

A Atenção Básica em Saúde (ABS) pode ser compreendida como um conjunto de práticas que abrange a promoção e manutenção da saúde, o diagnóstico social, a prevenção de agravos, a redução de danos, a cura e reabilitação de condições físicas/psíquicas/emocionais mais comuns, tendo como objetivos principais: ser a porta de entrada do usuário no sistema público de saúde, desenvolver ações com base territorial, atuar nos problemas mais frequentes da comunidade e desenvolver uma atenção integral que impacte na situação de saúde das pessoas e nos determinantes e condicionantes de saúde das coletividades ${ }^{(1)}$.

Esse nível de atenção apresenta uma série de atributos específicos, sendo os principais: prestação de serviços de primeiro contato; assunção de responsabilidade longitudinal pelo usuário; consideração do contexto e da dinâmica familiar; garantia de um cuidado integral; conhecimento das situações de saúde-adoecimento da população adscrita; e reconhecimento das diferentes necessidades dos grupos populacionais, bem como suas características étnicas, raciais e culturais ${ }^{(2)}$.

$\mathrm{Na}$ realidade brasileira, a ABS foi operacionalizada através da Estratégia de Saúde da Família (ESF). Em sua composição básica, é composta por um médico generalista, um enfermeiro, um ou dois técnicos de enfermagem, um dentista e seu auxiliar, um técnico em saúde bucal, um atendente social e um de regulação, quatro a seis agentes comunitários de saúde e, em algumas realidades, como em São Paulo e no Rio Grande do Sul, por alguns profissionais opcionais, como psicólogo e/ou assistente social ${ }^{(3)}$.

Criados com o intuito de dar um suporte técnico especializado aos profissionais das ESF e das equipes de ABS para populações específicas (consultórios na rua, equipes ribeirinhas e fluviais) surgiram os Núcleos de Apoio à Saúde da Família (NASF). Os profissionais a compor essas equipes devem compartilhar práticas e saberes em saúde, buscando auxiliá-las no manejo ou resolução de problemas clínicos e sanitários, bem como agregando práticas, na ABS, que ampliem o seu escopo de ofertas. Alguns dos profissionais que podem vir a fazer parte dessa equipe são: assistente social, educador físico, terapeuta ocupacional, psicólogo, entre outros ${ }^{(4)}$.

Com relação a esse último profissional, o seu modo de atuação deve ser pautado na produção de múltiplas formas de cuidado, na singularização do olhar e no respeito à diversidade cultural e subjetiva dos usuários, sem deixarse "escravizar" por rituais "mecanizados". Contudo, historicamente, os psicólogos têm atuado no contexto da Saúde Coletiva desconsiderando todos esses aspectos. Nesse sentido, revela-se a importância de se compreender como tem sido a atuação dos psicólogos no âmbito da ABS, uma vez que, no cotidiano de muitos desses profissionais, nem sempre são levados em consideração os princípios que norteiam esse nível de atenção ${ }^{(5)}$.

Dessa forma, tomando como pressupostos todos os aspectos discutidos até então, o presente trabalho objetivou 
identificar, nas evidências científicas, as atividades que caracterizam a atuação do psicólogo no contexto da Atenção Básica de Saúde.

\section{MÉTODOS}

O trabalho em questão trata-se de uma revisão sistemática, a qual pode ser compreendida como uma síntese rigorosa de todas as pesquisas relacionadas a uma temática específica. Esse tipo de estudo possui como princípios gerais a exaustão na busca dos estudos analisados, a seleção justificada por meio de critérios de inclusão e exclusão explícitos, a avaliação da qualidade metodológica, bem como a quantificação e posterior análise dos dados oriundos ${ }^{(6)}$.

A busca foi realizada entre os meses de agosto a dezembro de 2015, tomando como pressupostos para realização da revisão os definidos pelas recomendações do protocolo PRISMA. Este pode ser compreendido como um conjunto de 27 itens que auxiliam os pesquisadores no processo de elaboração de revisões sistemáticas através de instruções específicas para título, resumo, métodos, resultados, apoio financeiro, entre outras informações ${ }^{(7)}$.

Com relação aos procedimentos adotados, primeiramente, como uma maneira de possibilitar uma validade científica à procura realizada, foram utilizados os descritores "Psicologia", "Atuação" e "Atenção Básica" na Biblioteca Virtual de Saúde (BVS). Esses descritores também foram traduzidos para o espanhol e inglês. Em seguida, realizou-se uma busca direta nas bases de dados SciELO, LILACS e Index Psi Periódicos TécnicosCientíficos, utilizando as palavras-chave "Psicologia na Atenção Básica" como elemento norteador de investigação.

Posteriormente, foi elencada uma série de critérios de elegibilidade como definidores da análise das pesquisas, os quais foram: veículo de divulgação (periódicos indexados), período de publicação (entre os anos de 2010 a 2014), idioma (português, inglês ou espanhol), modalidade de produção (estudos empíricos, relatos de experiência e/ou estudos de casos) e qualidade metodológica (pesquisas que definissem precisamente quais eram seus participantes, instrumentos, procedimentos e tipo de estudo). Essa busca ocorreu a partir de uma análise minuciosa dos resumos das pesquisas encontradas, consistindo numa procura de estudos relacionados à atuação do psicólogo na Atenção Básica e que também se enquadrassem nos critérios de elegibilidade previamente determinados.

Esses procedimentos foram realizados por dois dos autores da referida pesquisa, primeiramente de maneira isolada, havendo em seguida uma confrontação dos dados de cada um, e posteriormente, um consenso entre ambos sobre os artigos a serem incluídos e excluídos da análise.

\section{RESULTADOS}

\section{Caracterização dos estudos}

A busca realizada $(n=107)$ identificou a presença de 67 estudos em Lilacs, 24 em Scielo e 16 em Index Psicologia, cujos resumos foram lidos detalhadamente e, em seguida, selecionados de acordo com os critérios de inclusão-exclusão, chegando-se ao final com um total de 11 pesquisas $^{(1,8-17)}$. Estas foram analisadas a partir de duas leituras detalhadas sobre cada texto: uma primeira de reconhecimento, e uma segunda com o intuito de identificar as atividades que caracterizam a atuação do psicólogo na ABS.

No Quadro I pode-se visualizar as pesquisas utilizadas na referida análise, bem como, a descrição sucinta de seus objetivos, métodos e principais resultados.

Desses artigos, quatro se propõem a estudar a atuação do psicólogo na ESF, outros quatro objetivam descrever a experiência de estágios em Saúde Coletiva, e três se propõem a retratar a experiência de psicólogos no NASF. Com relação aos tipos de estudo, sete são relatos de experiência, três são estudos empíricos e um é estudo de caso. Nesses estudos, a coleta de dados ocorreu por meio da utilização dos seguintes instrumentos: entrevista semiestruturada (3 estudos), grupo terapêutico (2), oficinas temáticas (1), prontuário da família (1), genograma dos usuários (1), roteiro de orientação para coleta de dados (1) e grupo operativo (1).

\section{Análise temática}

Os artigos foram divididos em duas categorias temáticas, de forma mutuamente excludente: (i) atividades realizadas na ESF, e (ii) atividades realizadas no NASF. Em cada uma delas foram contabilizadas as frequências de ações realizadas por profissionais de psicologia, tanto formados quanto em formação.

\section{DISCUSSÃO}

\section{Atividades realizadas na ESF}

Quando se organizam as ações do primeiro eixo temático, percebe-se o aparecimento das seguintes ações a serem desenvolvidas pelo psicólogo na ESF, a saber: reconhecimento do território de abrangência; ações grupais com a população adscrita; ações terapêuticas individualizadas; suporte técnico, assistencial e pedagógico à equipe de saúde; planejamento em equipe das ações a serem realizadas e função de preceptoria e de consultoria.

A literatura aponta que as ações a serem desenvolvidas pelos profissionais que compõem a ESF, independentemente de sua formação acadêmica devem ser: definição do território de atuação; atenção à saúde de acordo com as 


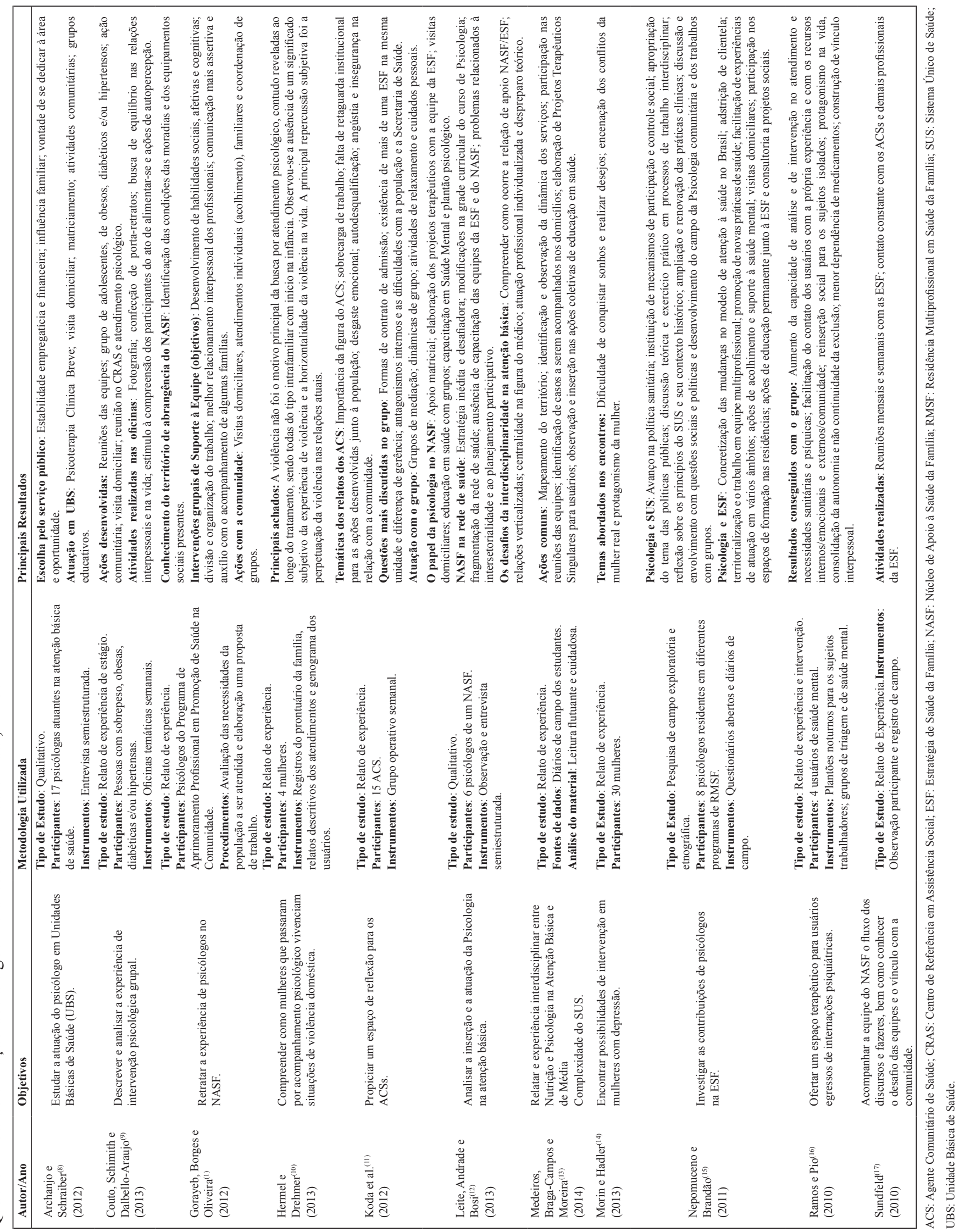


necessidades sócio-histórico-político-econômico-culturais da população; ações que priorizem grupos e fatores de riscos clínico-comportamentais, alimentares e/ou ambientais; acolhimento; atenção integral no cuidado; atividades em espaços comunitários; ações educativas; participação nos processos de gestão e no planejamento local de saúde e atenção domiciliar ${ }^{(18)}$.

Apesar de o psicólogo não estar instituído na Equipe de Saúde da Família, compreende-se, com base nos artigos analisados, que, quando presente nesse contexto, conforme é o caso de algumas realidades, como em São Paulo e no Rio Grande do Sul, esse profissional consegue realizar ações que estejam condizentes com os princípios de trabalho da ABS, os quais, conforme explicados anteriormente, são definidos como sendo o primeiro contato, a longitudinalidade, a abrangência, o arranjo da rede de saúde, a orientação para comunidade, a centralidade na família e a competência cultural $^{(2)}$.

Um aspecto interessante de ser notado é o aparecimento da atividade de apoio matricial. Trata-se de um arranjo organizacional que viabiliza proporcionar um suporte técnico especializado para as equipes responsáveis pelo desenvolvimento de ações básicas de saúde. Essas atividades são produzidas sob a forma de responsabilização pelos casos, que podem se efetivar através de discussões conjuntas dos casos, intervenções conjuntas junto às famílias e às comunidades, atendimentos conjuntos, e também na forma de supervisão e capacitação. No campo da saúde mental, são geralmente desenvolvidas pelo NASF e pelo Centro de Atenção Psicossocial (CAPS) ${ }^{(19)}$.

Um percalço perceptível na trajetória do psicólogo na ESF é a demanda de realize escuta individual, independentemente de onde ocorra a sua inserção, quando na realidade essa é uma necessidade técnica de todo profissional da área da saúde. Dessa forma, frequentemente, ele acaba atendendo a essa necessidade no seu cotidiano de trabalho; prova viva dessa realidade é o aparecimento das seguintes atividades durante a análise: plantão psicológico e atendimento psicológico (psicoterapia) ${ }^{(20)}$.

Sobre esses aspectos, é possível afirmar que a inserção dos psicólogos no contexto da Saúde Coletiva parece não ter modificado os modelos teóricos e práticos que fundamentam suas ações (ainda pautado na Psicologia Clínica Liberal), o que gera algumas dificuldades na realização de novas práticas voltadas para a produção social de saúde e da cidadania que estejam comprometidas com o bem-estar psicossocial dos indivíduos ${ }^{(5)}$.

\section{Atividades realizadas no NASF}

Ao sistematizar as atividades do segundo eixo temático, verificou-se, a partir da análise dos artigos, o aparecimento das seguintes atividades como possíveis de serem desenvolvidas pelos psicólogos no contexto do NASF: reconhecimento do território de abrangência; planejamento das ações com a ESF; ações de acolhimento; ações grupais; atendimento familiar; suporte técnico, assistencial e pedagógico às ESF e acionamento de outros dispositivos da rede como o Sistema Único de Assistência Social (SUAS).

Nesse sentido, a partir da leitura dos eixos encontrados, percebe-se que o atendimento clínico individualizado não é suficiente para atender a demanda real dos serviços os quais o NASF presta assistência, uma vez que tal modalidade interventiva não gera uma transformação social. Dessa forma, o psicólogo necessita cada vez mais reconfigurar sua prática e tornar-se um "sujeito dinamizador", alinhando suas ações aos fundamentos políticos-ideológicos do sistema de saúde vigente ${ }^{(5,21)}$.

Portanto, a atuação psicológica nesse núcleo, assim como dos demais profissionais, deve ser norteada a partir de oito diretrizes - interdisciplinaridade, intersetorialidade, territorialização, integralidade, educação permanente em saúde, humanização, controle social e promoção da saúde -, podendo atuar em oito áreas estratégicas: atividade física/ práticas corporais; práticas integrativas e complementares; reabilitação; alimentação e nutrição; saúde mental; serviço social; saúde da criança, do adolescente e do jovem; saúde da mulher e assistência farmacêutica ${ }^{(22)}$.

De acordo com as cartilhas governamentais, o apoio oferecido pelo NASF pode ocorrer, por exemplo, através de consultas conjuntas entre o psicólogo e o médico da ESF, ou ainda através de consultas com o psiquiatra, pactuadas previamente com a Equipe de Saúde da Família como parte do projeto terapêutico de determinado usuário. Portanto, as atividades a serem desenvolvidas podem englobar ações realizadas somente pelos profissionais do NASF, ou destes em conjunto com as equipes vinculadas, ou, ainda, somente pelos profissionais da ESF, depois de um suporte técnico especializado $^{(4)}$.

Esse apoio técnico especializado também pode ocorrer através do apoio matricial, talvez a atividade mais desempenhada pelo psicólogo nesse contexto. Contudo, apesar do matriciamento ser um norteador de trabalho nesse dispositivo de saúde, frequentemente muitos profissionais desempenham essa atividade de uma forma bastante restrita, principalmente, através tão somente de encaminhamentos de casos, o que por si só não gera um suporte técnico a $\mathrm{ESF}^{(11)}$.

Por fim, outro aspecto importante a ser ressaltado refere-se à coordenação da rede de atenção à saúde, contudo, ainda são grandes os desafios para que ela assuma esse papel. Dentre os "nós críticos" apontados para explicar essa realidade, destacam-se a falta de política institucional 
direcionada ao fortalecimento da Atenção Básica; a representação social das comunidades sobre esse ponto de atenção; a falta de legitimidade social; a visão restrita de gestores que tendem a compreendê-la, por vezes, como seletiva, além da insuficiência de recursos qualificados para um modus faciendi dessa estratégia, o que resulta numa notável carência de dispositivos de apoio e logística que favoreçam a inovação das práticas, o acolhimento dos usuários e a vinculação dos mesmos na atenção básica ${ }^{(23)}$.

\section{CONCLUSÃO}

As evidências científicas dos últimos cinco anos (2010-2014) abordam com muita pertinência a atuação do psicólogo no contexto da Atenção Básica em Saúde, uma vez que foi perceptível a concretização de práticas que levam em consideração tanto aspectos micropolíticos, referentes à subjetividade, quanto aspectos macroestruturais, alusivo aos aspectos sócio-histórico-econômico-político-culturais.

\section{REFERÊNCIAS}

1. Gorayeb R, Borges C, Oliveira CM. Psicologia na atenção primária: ações e reflexões em programa de aprimoramento profissional. Psicol Ciênc Prof. 2012;32(3):674-85.

2. Giovanella L, Mendonça MHM. Atenção primária em Saúde. In: Giovanella L. Políticas e sistemas de saúde no Brasil. Rio de Janeiro: FIOCRUZ; 2008.p.575-625.

3. Gil CRR. Atenção primária, atenção básica e saúde da família: sinergias e singularidades do contexto brasileiro. Cad Saúde Pública. 2006;22(6):1171-81.

4. Ministério da Saúde (BR), Secretária de Atenção à Saúde, Departamento de Atenção Básica. Núcleo de Apoio a Saúde da Família: ferramentas para a gestão e para o trabalho cotidiano. Brasília: Ministério da Saúde; 2014.v.1.

5. Dimenstein M. O psicólogo e o compromisso social no contexto da saúde coletiva. Psicol Estud. 2001;6(2):5763.

6. Galvão CM, Sawada NO, Trevisan MA. Revisão Sistemática: Recurso que proporciona a incorporação das evidências na prática da enfermagem. Rev Latinoam Enferm. 2004;12(3):549-56.

7. Costa LOP, Maher CG, Lopes AD, Noronha MA, Costa LCM. Como escrever de forma transparente artigos científicos relevantes para a prática da Fisioterapia. Rev Bras Fisioter. 2011;15(4):267-71.
8. Archanjo AM, Schraiber LBA. A Atuação dos Psicólogos em Unidades Básicas de Saúde na cidade de São Paulo. Saúde Soc. 2012;21(2):351-63.

9. Couto LLM, Schimith PB, Dalbello-Araujo M. Psicólogo em Ação no SUS: a Interdisciplinaridade Posta à Prova. Psicol Ciênc Prof. 2013;33(2):500-11.

10. Hermel JS, Drehmer LBR. Repercussões da violência intrafamiliar: Um estudo com mulheres em acompanhamento psicológico. Psicol Argum. 2013;31(74):437-46.

11. Koda MY, Silva DV, Machado MAS, Naldos SMS. Grupo com agentes comunitárias: a construção de novas possibilidades do cuidar. Psicol Ciênc Prof. 2012;32(2):506-15.

12. Leite DC, Andrade AB, Bosi MLMA. A inserção da psicologia nos núcleos de apoio à saúde da família. Physis (Rio J). 2013;23(4):1167-87.

13. Medeiros MAT, Braga-Campos FC, Moreira MIB. A integralidade como eixo da formação em proposta interdisciplinar: estágios de Nutrição e Psicologia no campo da Saúde Coletiva. Rev Nutr. 2014;27(6):78598.

14. Morin PV, Hadler OH. Mosaico de vidas: reflexões sobre o sociopsicodrama na saúde coletiva. Rev Bras Psicodrama. 2013;21(1):55-66.

15. Nepomuceno LB, Brandão IR. Psicólogos na Estratégia de Saúde da Família: Caminhos Percorridos e Desafios a Superar. Psicol Ciênc Prof. 2011;31(4):762-77.

16. Ramos PF, Pio DAM. Construção de um projeto de cuidado em saúde mental na atenção básica. Psicol Ciênc Prof. 2010;30(1):212-23.

17. Sundfeld AC. Clínica ampliada na atenção básica e processos de subjetivação: relato de uma experiência. Physis (Rio J). 2010;20(4):1079-97.

18. Ministério da Saúde (BR), Secretaria de Atenção à Saúde, Departamento de Atenção Básica. Política Nacional de Atenção Básica. Brasília: Ministério da Saúde; 2012.

19. Dimenstein M, Severo AK, Brito M, Pimenta AL, Medeiros V, Bezerra E. O apoio matricial em unidades de saúde da família: experimentando inovações em saúde mental. Saúde Soc. 2009;18(1):63-74.

20. Meira MA, Silva MO. Atuação da Psicologia na Estratégia Saúde da Família: a Experiência de um Psicólogo em uma Residência Multiprofissional. Rev Bras Ciênc Saúde. 2011;15(3):369-76. 
21. Azevedo NS, Kind L. Psicologia nos Núcleos de Apoio à Saúde da Família em Belo Horizonte. Psicol Ciênc Prof. 2013;33(3):520-35.

22. Mângia EF, Lancman S. Núcleos de Apoio à Saúde da Família: integralidade e trabalho em equipe multiprofissional. Rev Ter Ocup. 2008;19(2):i.
23. Rodrigues LBB, Silva PCS, Peruhype RC, Palha PF, Popolin MP, Crispim JA, et al. A atenção primária à saúde na coordenação das redes de atenção: uma revisão integrativa. Ciên Saúde Coletiva. 2014;19(2):343-52.

\section{Endereço para correspondência:}

Matheus Barbosa Rocha

Universidade Federal do Piauí - UFPI

Curso de Psicologia

Campus Universitário Ministro Petrônio Portella, s/n Bairro: Ininga

CEP 64049-550 - Teresina - PI - Brasil

E-mail: matheusbr.psico@live.com 